Article

\title{
Pseudomonas protegens FJKB0103 Isolated from Rhizosphere Exhibits Anti-Methicillin-Resistant Staphylococcus aureus Activity
}

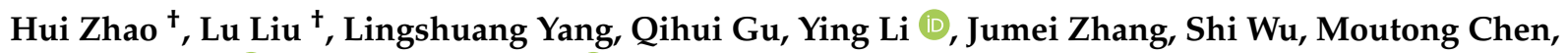 \\ Xinqiang $\mathrm{Xie}^{*}$ (D) and Qingping $\mathrm{Wu} *$ (D)
}

check for updates

Citation: Zhao, H.; Liu, L.; Yang, L.; Gu, Q.; Li, Y.; Zhang, J.; Wu, S.; Chen, M.; Xie, X.; Wu, Q. Pseudomonas protegens FJKB0103 Isolated from Rhizosphere Exhibits

Anti-Methicillin-Resistant Staphylococcus aureus Activity.

Microorganisms 2022, 10, 315.

https://doi.org/10.3390/

microorganisms10020315

Academic Editor: Chu-Young Kim

Received: 26 December 2021

Accepted: 19 January 2022

Published: 28 January 2022

Publisher's Note: MDPI stays neutral with regard to jurisdictional claims in published maps and institutional affiliations.

Copyright: (C) 2022 by the authors. Licensee MDPI, Basel, Switzerland. This article is an open access article distributed under the terms and conditions of the Creative Commons Attribution (CC BY) license (https:// creativecommons.org/licenses/by/ $4.0 /)$.

\author{
Guangdong Provincial Key Laboratory of Microbial Safety and Health, State Key Laboratory of Applied \\ Microbiology Southern China, Institute of Microbiology, Guangdong Academy of Sciences, \\ Guangzhou 510070, China; zhaohuichinese@163.com (H.Z.); luckyliulululu@163.com (L.L.); \\ yangls8272@163.com (L.Y.); guqh888@163.com (Q.G.); liying@gdim.cn (Y.L.); zhangjm926@126.com (J.Z.); \\ wushiloveyou@126.com (S.W.); cmtoon@hotmail.com (M.C.) \\ * Correspondence: woshixinqiang@126.com (X.X.); wuqp203@163.com (Q.W.) \\ + These authors contributed equally to this work.
}

\begin{abstract}
Staphylococcus aureus is amongst the most virulent pathogens, causing chronic and lifethreatening human infections. Methicillin-resistant S. aureus (MRSA) are multidrug-resistant strains, and the ability of forming a biofilm reduces their sensitivity to antibiotics. Thus, the alternative compounds inhibiting both resistant strains and biofilm formation are in high demand. In our study, the strain FJKB0103 was isolated from the rhizosphere of Garcinia mangostana, showing strong anti-MRSA activity. We performed molecular phylogenic analysis, analyzed average nucleotide identity (ANI), in silico DNA-DNA hybridization (isDDH), and biochemical characteristics to identify strain FJKB0103 as Pseudomonas protegens. Herein, the genome of strain FJKB0103 was sequenced and subjected to antiSMASH platform, mutational, and functional analyses. The FJKB0103 draft genome was $6,776,967 \mathrm{bp}$ with a $63.4 \% \mathrm{G}+\mathrm{C}$ content, and 16 potential secondary compound biosynthetic clusters in P. protegens FJKB0103 were predicted. The deletion mutant and complementary analysis suggested that DAPG was the anti-MRSA compound. Further tests showed that MRSA strains were sensitive to DAPG, and the lysis of bacterial cells was observed at a high concentration of DAPG. Additionally, DAPG inhibited the biofilm formation of MRSA at subinhibitory concentration. These results suggested that DAPG might be a good alternative treatment to control infections caused by MRSA.
\end{abstract}

Keywords: Staphylococcus aureus; Pseudomonas; DAPG; biofilm

\section{Introduction}

Staphylococcus aureus is a common opportunistic Gram-positive pathogen that has ubiquitous distribution in nature, such as on the skin and mucosae of the human population, food, air, and water. S. aureus can cause wound infections and toxin-mediated syndromes as well as systemic and life-threatening diseases [1]. Antibiotic application is the major method to control an $S$. aureus infection, but the extensive use and misuse of antibiotics in both human and animal medicine have led to an escalating challenge with multidrug-resistant bacterial strains, especially Methicillin-resistant S. aureus (MRSA) [2]. MRSA was first reported in the 1960s [3], and it has been recognized as a major cause of healthcare-associated infections worldwide due to its multidrug resistance to almost all the currently available antibiotics except vancomycin and teicoplanin [4]. Furthermore, biofilm-associated bacteria show resistance to antibiotics: biofilm matrix protects the bacteria from antibiotics by limiting their diffusion or repulsion, and cells within the biofilm, particularly those deep within the matrix, exist in a slow-growing state, which can limit the 
efficacy of antibiotics, especially those that target active cell processes [5]. Therefore, new treatment strategies affecting both resistant strains and bacterial biofilm formation are in crucial demand [5].

Pseudomonas is a diverse genus of $\gamma$-proteobacteria with more than 200 validly named species exhibiting varied lifestyles in a wide range of environments, including soil, water, plant surfaces, and animals (https://lpsn.dsmz.de/genus/pseudomonas, accessed on 20 November 2021). The complex ecological environments of Pseudomonas spp. promote genomic diversity. The bacteria acquire and discard genomic fragments in the process of developing a genetic repertoire customized to their special niche, especially the biosynthetic gene cluster of secondary metabolites. Pseudomonas spp. have received much attention in recent decades due to the production of a remarkable array of medically and agriculturally important secondary metabolites, including 2,4-diacetylphloroglucinol (DAPG), nonribosomal peptide synthase (NRPS), pyrrolnitrin (PRN), and pyoluteorin (PLT), all of which have been proved to have antimicrobial activity [6]. DAPG could suppress a wide variety of plant pathogens, including bacteria, fungi, and omycetes [7]. The main antimicrobial mechanism of DAPG is an alteration of the cell membrane leading to the dissipation of the proton gradient across the biological membranes, disorganization of hyphal tips, including alteration of the plasma membrane, vacuolization and cell content disintegration, and inhibition of zoosporogenesis, motility, and germination of zoospore [810]. DAPG is a phloroglucinol derivative and is synthesized by phl biosynthetic gene cluster (BCG) that comprises nine genes such as phlA, phlC, phlB, phlD, phlE, phlF, phlG, $p h l H$, and phll. Four genes (phlABCD) are directly involved in DAPG biosynthesis [11]. Type III polyketide synthase (encoded by gene phlD) catalyzes the condensation of three malonyl-CoA molecules to produce phloroglucinol (PG) [12], and the multimeric acetyltransferase complex Phl(A2C2)2B4 (encoded by gene phlACB) mediates the acylation of PG to form monoacetylphloroglucinol (MAPG) and of MAPG to form DAPG [13]. PhlE is involved in the transport of DAPG [14]. Gene phlF encodes a TetR family transcriptional regulator that represses DAPG production [15]. Gene phlG that codes for a DAPG special hydrolase is regulated by another TetR regulatory protein $\mathrm{PhlH}$ [16].

S. aureus Sta24-1 is one of the prevalent MRSA strains in China and is resistant to more than ten antibiotics [17]. In the present work, we reported a novel isolate FJKB0103 that inhibited the growth of MRSA strain Sta24-1. The taxonomic status of strain FJKB0103 was determined by phylogenetic analyses, genomic analysis, and phenotypic characteristics. The anti-MRSA compound was identified by genome mining and genetic analysis. The data we obtained will enrich the compound database that might be used as an alternative to control infections caused by MRSA.

\section{Materials and Methods}

\subsection{Strains and Growth Conditions}

Bacterial strains and plasmids used in this study are listed in Supplementary Table S1. Strain FJKB0103 and its mutants were grown in Luria-Bertani broth (LB) or King's B medium (Proteose peptone No. 3, $10 \mathrm{~g} ; \mathrm{K}_{2} \mathrm{HPO}_{4}, 1.5 \mathrm{~g} ; \mathrm{MgSO}_{4} \cdot 7 \mathrm{H}_{2} \mathrm{O}, 1.5 \mathrm{~g}$; Mannitol, 10 g; $\mathrm{KB})$ at $28^{\circ} \mathrm{C}$. Escherichia coli DH5 $\alpha$ was grown at $37^{\circ} \mathrm{C}$ in LB medium. Staphylococcus aureus, Listeria monocytogene, and Bacillus cereus were grown at $37^{\circ} \mathrm{C}$ in LB or PDA medium. Where indicated, media was supplemented with ampicillin $(50 \mu \mathrm{g} / \mathrm{mL})$ or kanamycin $(50 \mu \mathrm{g} / \mathrm{mL})$ and/or 5-bromo-4-chloro-3-indolyl-D-galactopyranoside (X-Gal; $40 \mu \mathrm{g} / \mathrm{mL}$ ).

\subsection{Isolation and Assays for Antagonistic Capacity of Strain FJKB0103}

The soil samples were collected from the rhizosphere of Garcinia mangostana in Zhangzhou, China. Five grams of soil sample suspended in $30 \mathrm{~mL}$ of sterile water was incubated at $28^{\circ} \mathrm{C}$ with shaking at $140 \mathrm{rpm}$. After $4 \mathrm{~h}$, the suspension was serially diluted with sterile water, and $100 \mu \mathrm{L}$ of the diluted soil suspension was spread onto KB agar. The individual colonies with different colors or morphology were selected for further purification by streaking onto fresh LB agar. For antibacterial assays, $5 \mu \mathrm{L}$ of an overnight 
culture of isolated strains were dropped onto the PDA plate center, and after inoculating at $28{ }^{\circ} \mathrm{C}$ for 2 days, the LB mixed with $10 \%$ saturated pathogenic bacteria was poured on the PDA plate, inoculated at $37^{\circ} \mathrm{C}$ for $12 \mathrm{~h}$.

\subsection{Genome Sequencing and Annotation}

The genomic DNA of FJKB0103 was extracted by using a DNA purification kit (Magen, Guangzhou, China) according to the manufacturer's protocols. The paired-end library was used to sequence on the Illumina Miseq 550 platform. De novo genome assembly was performed using SPAdes pipeline version 3.12, and sequences shorter than $200 \mathrm{bp}$ were trimmed. The draft genomes of FJKB0103 were annotated by Prokka 1.13.7, coding sequences were identified and annotated using Prodigal, rRNAs were predicted by RNAmmer, and tRNAs and noncoding RNAs were predicted using Aragorn and Infernal, respectively.

\subsection{Identification of Strain FJKB0103 Based on Genome}

The 16S rRNA gene was amplified using primers 27F and 1492R (Table S2). The result sequence was deposited to the EzBioCloud database (https://www.ezbiocloud. net, accessed on 25 October 2021) for preliminary identification based on the sequence similarity values between strain FJKB0103 and the related species. The genes 16S rRNA, gyrB, rpoB, and rpoD of Pseudomonas species closely related to strain FJKB0103 based on $16 \mathrm{~S}$ rDNA similarities were retrieved from their GenBank depositions or their whole genome sequences. The multiple sequence alignments were made by using CLUSTAL_W software. Phylogenetic trees were reconstructed using both neighbor-joining and the maximum likelihood methods by Mega 5 [18]. Bootstrap analysis was performed using 1000 replications. Average nucleotide identity (ANI) values were determined by using the Orthologous Average Nucleotide Identity Tool (OAT) (http: / / www.ezbiocloud.net/ sw / oat, accessed on 28 October 2021) [19], and the two ANI scores, ANI algorithm using blast (ANIb), and ANI of orthologous genes (OrthoANI) were calculated between strain FJKB0103 and the type strains of the closely related Pseudomonas species. The Genome-toGenome Distance Calculator (GGDC) was employed for in silico DNA-DNA hybridization (is DDH) analysis, and all three equations in the GGDC program were used (version 2.1; http:/ /ggdc.dsmz.de/distcalc2.php, accessed on 29 October 2021) [20].

\subsection{Genome Analysis of Putative Secondary Metabolite Clusters}

The Antibiotics and Secondary Metabolite Analysis Shell pipeline (antiSMASH) was used to analyze the secondary metabolite gene clusters of strain FJKB0103 [21]. Briefly, the detection strictness was "relaxed", and all "Extra Features", including KnownClusterBlast, ClusterBlast, SubClusterBlast, Cluster Pfam analysis, Pfam-based GO term annotation, and ActiveSiteFinder, were employed for biosynthetic gene cluster (BGC) border prediction and analysis.

\subsection{Construction of P. protegens FJKB0103 Deleted Mutants and Complementary Strain}

To confirm the effect of predicted gene clusters on antibiotic activity, the gene clusters, of which the similarity was more than $90 \%$, were selected for further study. We constructed in-frame deleted mutants of gene clusters encoding DAPG, PRN, PLT, and orfamide as in previous reports, respectively [16]. An in-frame deletion of the phlD gene involved in the biosynthesis of DAPG was made using a two-step homologous-recombination strategy. Briefly, the upstream and downstream fragments of phlD gene were PCR amplified using the primer pairs listed in Table S2. The PCR fragments were digested with restriction enzymes and then cloned into the suicide vector $\mathrm{p} 2 \mathrm{P} 24-\mathrm{Km}$ to generate plasmid p2P24- $\Delta \mathrm{phlD}$. The suicide plasmid p2P24- $\Delta$ phlD was introduced into strain FJKB0103 by electroporation. Colonies that appeared on LB plates containing kanamycin were picked up and inoculated in liquid LB medium without antibiotics. Overnight cultures were then plated on LB agar supplemented with $20 \%$ sucrose to generate the mutants, and the mutants were confirmed 
by PCR. The same approach was used to construct in-frame deletion mutants of the prnA, plt $A$, and orf $A$ genes, yielding the mutants $\triangle \mathrm{prnA}, \Delta \mathrm{pltA}$, and $\triangle$ orfA, respectively (Table S1). For complementary analysis, the DAPG biosynthetic gene cluster phlACBD was amplified using primers phl-2425-HindIII and phl-7049-KpnI and inserted into the shuttle vector pRK415, yielding the complementary plasmid pRK415-phl. The resultant plasmid was introduced into phlD gene deletion mutant to generate complementary strain $\Delta$ phlD-C. Mutants and complementary strains were tested for their abilities to inhibit $S$. aureus.

\subsection{Extraction and Detection of DAPG}

The extraction and detection of DAPG were performed according to a previous method [22]. P. protegens FJKB0103 and its mutants were grown in KB broth for $48 \mathrm{~h}$ at $28{ }^{\circ} \mathrm{C}$. Samples $(800 \mu \mathrm{L})$ were acidified with $100 \mu \mathrm{L}$ of $10 \%$ trifluoroacetic acid (TFA) and extracted with $900 \mu \mathrm{L}$ of ethyl acetate. The organic phase containing DAPG was evaporated to dryness and suspended in $50 \mu \mathrm{L}$ methanol. Volumes of the extracts of $10 \mu \mathrm{L}$ were detected by an Agilent 1260 system equipped with a C18 column $(4.6 \times 150 \mathrm{~mm})$. The samples were eluted from the column using a gradient of $55 \%$ acetonitrile: $45 \%$ water containing $0.1 \%$ phosphoric acid over $8 \mathrm{~min}$ at a flow rate of $1.0 \mathrm{~mL} / \mathrm{min}$. DAPG was monitored at $270 \mathrm{~nm}$.

\subsection{Determination of the Minimum Inhibitory Concentration (MIC) and Minimum Bactericidal} Concentration (MBC) of DAPG, MAPG, PG, or Vancomycin on S. aureus

The values of MIC were evaluated following the CLSI guideline: suspension of S. aureus strain with $0.5 \mathrm{McF}$ arland was prepared by making a direct broth suspension of isolated colonies selected from a $24 \mathrm{~h}$ agar plate; the suspension was diluted as 1:300 into $2 \mathrm{~mL}$ of cation-adjusted Muller Hinton broth (CAMHB), of which the final inoculum was approximate to $5 \times 10^{5} \mathrm{CFU} / \mathrm{mL} ; 10 \mu \mathrm{L}$ DAPG, MAPG, PG, and vancomycin (various concentrations) were added to a tube, respectively; the MIC was the concentration of the drug that resulted in no visible bacterial growth after $20 \mathrm{~h}$ incubation at $37^{\circ} \mathrm{C}$ in an ambient air incubator [23]. Content of the tube with no visible growth was spread on LB agar and incubated for $24 \mathrm{~h}$ at $37^{\circ} \mathrm{C}$. LB agar with the lowest concentration and no bacterial growth was scored as MBC [24].

\subsection{Bacteriolytic Assay}

The bacteriolytic activity of DAPG to MRSA bacterial cells was evaluated as in a previous report [25]. Briefly, the S. aureus Sta24-1 strain was grown in tryptic soy broth (TSB) overnight at $37^{\circ} \mathrm{C}, 180 \mathrm{rpm}$. The overnight culture of S. aureus Sta24-1 was diluted as 1:100 into $2 \mathrm{~mL}$ of TSB. S. aureus Sta24-1 was exposed to DAPG with different concentrations at the early stage of exponential growth, and the negative control (CK) was exposed to methanol. The optical density $\left(\mathrm{OD}_{600}\right)$ was measured at $0,6,18$, and $24 \mathrm{~h}$ after incubation with DAPG.

\subsection{Biofilm Formation and Swarming Motility}

The effect of subinhibitory levels of DAPG on S. aureus biofilm formation were detected as in a previous study. S. aureus culture was diluted as 1:100 into $3 \mathrm{~mL}$ TSB glass tubes, and $3 \mu \mathrm{L}$ of DAPG at subinhibitory level was added and incubated at $37^{\circ} \mathrm{C}$ stationary. After $24 \mathrm{~h}$ of incubation, the medium was removed by pipetting, and tubes were gently washed with sterile water, biofilms on the inner surface of glass tubes were stained with $0.1 \%$ crystal violet and quantified at $570 \mathrm{~nm}$ [26]. The swarming motility of $P$. protegens FJKB0103 and its mutants was studied by spotting $5 \mu \mathrm{L}$ of an overnight cell suspension on soft $(0.3 \% w / v)$ LB agar and evaluating surface swarming motility after incubation at $28^{\circ} \mathrm{C}$ for $24 \mathrm{~h}$ [27]. 


\section{Results}

\subsection{Antibiotic Activity of Isolates from Rhizosphere}

Thirty-seven bacterial strains were isolated from the rhizosphere of Garcinia mangostana. All isolates were tested for their antibiotic activities against methicillin-resistant Staphylococcus aureus (MRSA) Sta24-1. A total of five isolates showed antibacterial ability against MRSA Sta24-1, including the strain FJKB0103, whose anti-MRSA activity was the best (Figure S1). Moreover, the strain FJKB0103 exhibited broad-spectrum antibiotic activities against food-borne pathogens like Listeria monocytogenes and Bacillus cereus, but not Escherichia coli (Figure S1).

\subsection{General Genome Characteristics of Strain FJKB0103}

The size of strain FJKB0103 draft genome was 6,776,967 bp with a G + C content of $63.4 \%$. It consisted of 40 contigs. A total of 6170 genes were predicted, which included 6096 coding sequences (CDSs) and 74 RNA genes including 7 rRNA genes, 66 tRNAs genes, and one tmRNA gene. The general features of the strain FJKB0103 genome are summarized in Table 1.

Table 1. Genome statistics for P. protegens FJKB0103.

\begin{tabular}{cc}
\hline Features & Chromosome \\
\hline Number of contigs & 40 \\
Size (bp) & $6,776,967$ \\
G + C content & $63.4 \%$ \\
Number of genes & 6170 \\
Number of CDSs & 6096 \\
Number of rRNAs & 7 \\
Number of tRNAs & 66 \\
Number of tmRNAs & 1 \\
\hline
\end{tabular}

\subsection{Identification of Strain FJKB0103 Based on Polyphasic Taxonomy}

The 16S rRNA gene sequence of strain FJKB0103 showed the highest similarities to P. protegens $\mathrm{CHA0}{ }^{\mathrm{T}}$ and $P$. saponiphila DSM $9751^{\mathrm{T}}$, which are members of the $P$. fluorescens group. A phylogenetic tree based on $16 \mathrm{~S}$ rDNA and multilocus sequence analysis (MLSA) of the housekeeping genes (16S rRNA, $g y r B, r p o B$ and $r p o D)$ were built with 28 Pseudomonastype strains, and Cellvibrio japonicus DSM $16015^{\mathrm{T}}$ was selected as an outgroup $(\mathrm{n}=30)$. Both the phylogenetic trees showed that strain FJKB0103 formed a monophyletic cluster with P. protegens subgroup strains including P. protegens $\mathrm{CHA} 0^{\mathrm{T}}$ and P. saponiphila DSM9751 ${ }^{\mathrm{T}}$, and the bootstrap values were high (Figures 1 and S2). These data indicated that strain FJKB0103 belonged to the $P$. protegens subgroup within the $P$. fluorescens lineage.

To more accurately determine the phylogenetic position of strain FJKB0103, ANI and is DDH analysis were performed based on genomic sequence. The ANI values between FJKB0103 and P. protegens strains that included type strain $P$. protegens $\mathrm{CHA} 0^{\mathrm{T}}$ were higher than the threshold range (95-96\%) for species delineation (Table 2). Furthermore, three isDDH values were also higher than $70 \%$ cut-off within these species. Both ANI and isDDH scores were lower than the values for species delineation between FJKB0103 and other Pseudomonas type strains (Table 2). The high similarity between FJKB0103 and CHA0 ${ }^{\mathrm{T}}$ was also confirmed at the biochemical level using API 50CH (bioMérieux, France; Table S3). All these data demonstrated that the strain FJKB0103 belonged to P. protegens. 


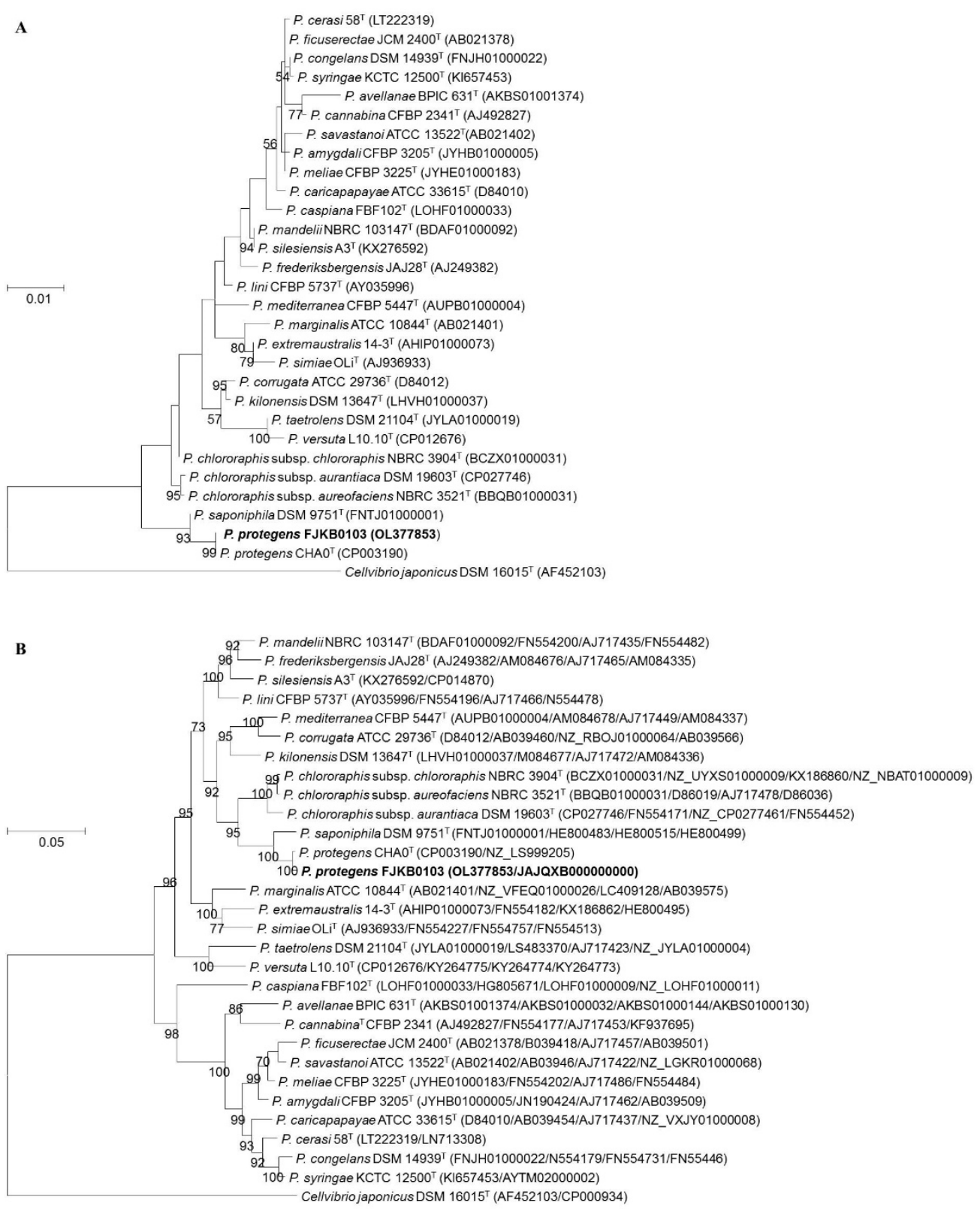

Figure 1. (A) Maximumlikelihood phylogenetic tree based on 16S rRNA gene sequences showing the relationship of strain FJKB0103 to the closely related strains. GenBank accession numbers of $16 \mathrm{~S}$ rRNA genes are given in parentheses. (B) Maximum likelihood phylogenetic tree based on MLSA (16S rRNA, gyrB, rpoD, and $r p o B$ ) showing the relationship of strain FJKB0103 to the closely related strains. The sequences of their genes 16S rRNA (1377 bp), gyrB (734 bp), rpoB (915 bp) and rpoD (723 bp) were retrieved from their GenBank depositions or from their whole-genome sequences. GenBank accession numbers are given in parentheses (accession numbers are given in parentheses in the following order: $16 \mathrm{~S}$ rRNA, gyrB, rpoB, and rpoD genes. Cellvibrio japonicas DSM $16015^{\mathrm{T}}$ was used as an outgroup. Numbers at nodes are bootstrap values $(\geq 50 \%)$ from 1000 repetitions. The strain FJKB0103 was highlighted in bold. ${ }^{\mathrm{T}}$ means type strain. 
Table 2. Average nucleotide identity (ANI) and in silico DNA-DNA hybridization (isDDH) values between the genome sequence of strain FJKB0103 and closely related Pseudomonas species.

\begin{tabular}{|c|c|c|c|c|c|}
\hline \multirow{2}{*}{ Species } & \multicolumn{2}{|c|}{ ANIb (\%) ${ }^{a}$} & \multicolumn{3}{|c|}{ isDDH (\%) ${ }^{b}$} \\
\hline & ANIb & OrthoANI & Formula 1 & Formula 2 & Formula 3 \\
\hline $\begin{array}{l}\text { P. protegens Cab57 } \\
\text { (AP014522.1) }\end{array}$ & 98.44 & 98.51 & 96.80 & 86.90 & 97.10 \\
\hline $\begin{array}{l}\text { P. protegens CHA0 }{ }^{\mathrm{T}} \\
\text { (LS999205.1) }\end{array}$ & 98.13 & 98.27 & 95.10 & 84.00 & 95.60 \\
\hline $\begin{array}{l}\text { P. protegens H78 } \\
\text { (CP013184.1) }\end{array}$ & 98.09 & 98.21 & 94.00 & 83.70 & 94.70 \\
\hline $\begin{array}{l}\text { P. protegens SN15-2 } \\
\quad(\text { CP043179.1) }\end{array}$ & 98.01 & 98.09 & 92.10 & 83.00 & 93.10 \\
\hline P. protegens & & & & & \\
\hline $\begin{array}{l}\text { FDAARGOS_307 } \\
\text { (CP022097.2) }\end{array}$ & 98.00 & 98.11 & 92.10 & 83.00 & 93.10 \\
\hline $\begin{array}{l}\text { P. protegens Pf-5 } \\
\text { (NC_004129.6) }\end{array}$ & 97.98 & 98.11 & 92.10 & 83.00 & 93.10 \\
\hline $\begin{array}{l}\text { P. saponiphila DSM } 9751^{\mathrm{T}} \\
\text { (FNTJ00000000.1) }\end{array}$ & 89.30 & 89.71 & 62.90 & 38.60 & 57.50 \\
\hline P. cerasi $58^{\mathrm{T}}(\mathrm{LT} 222319.1)$ & 77.25 & 78.07 & 19.60 & 22.50 & 19.10 \\
\hline $\begin{array}{l}\text { P. meliae CFBP } 3225^{\mathrm{T}} \\
\text { (JYHE00000000.1) }\end{array}$ & 77.23 & 77.96 & 19.10 & 22.50 & 18.70 \\
\hline $\begin{array}{l}\text { P. congelans DSM } 14939^{\mathrm{T}} \\
(\mathrm{NJH} 0000000)\end{array}$ & 77.13 & 78.10 & 19.50 & 22.40 & 19.10 \\
\hline $\begin{array}{l}\text { P. tremae ICMP9151 } \\
\text { (LJRO00000000.1) }\end{array}$ & 76.67 & 77.50 & 18.10 & 22.30 & 17.80 \\
\hline $\begin{array}{l}\text { P. caspiana FBF102 }{ }^{\mathrm{T}} \\
\text { (LOHF00000000.1) }\end{array}$ & 76.45 & 77.20 & 17.80 & 22.60 & 17.60 \\
\hline
\end{tabular}

a The average nucleotide identity (ANI) (ANIb and OrthoANI) was calculated using OAT software; ${ }^{\mathrm{b}}$ The isDDH values were calculated using the Genome-to-Genome Distance Calculator (http:/ /ggdc.dsmz.de/distcalc2.php, accessed on 29 October 2021$) ;{ }^{\mathrm{T}}$ means type strain.

\subsection{Genome Mining to Reveal the Putative Secondary Metabolite Clusters}

To predict putative secondary metabolites, such as antibiotics, bacteriocin, and NRPS genes, the genome sequence was analyzed using antiSMASH platform version 5.0. Sixteen secondary compound biosynthetic gene clusters (BGCs) were predicted in P. protegens FJKB0103 (Tables 3 and S4), including DAPG, PRN, PLT, and NRPS, which showed antibiotic activity in the previous study [6]. To uncover the specific secondary metabolites, the secondary compound BGCs of several genome complete P. protegens strains Cab57, CHA0 T, H78, SN15-2, FDAARG, and Pf-5, and six closely related type strains P. saponiphila DSM9751 T, P. cerasi $58^{\mathrm{T}}$. P. meliae CFBP3225 $\mathrm{T}$, P. congelans DSM14939 $\mathrm{T}$, P. tremae ICMP9151 ${ }^{\mathrm{T}}$, and P. caspiana $\mathrm{FBF} 102^{\mathrm{T}}$ were predicted and compared with FJKB0103 secondary compound BGCs. Strain FJKB0103 had the largest BGC numbers, while P. caspiana FBF102 ${ }^{\mathrm{T}}$ had the fewest (Table 3). NRPSs production was the only common feature presented in all strains, whereas DAPG, PRN, PLT, and orfamide BGCs were conserved in all the $P$. protegens strains (Table 3 ). The results indicated that DAPG, PRN, PLT, and orfamide might be the specific compounds for P. protegens species, which might be involved in the antibiotic activity of $P$. protegens FJKB0103. 
Table 3. Secondary metabolite and antibiotic gene clusters in P. protegens FJKB0103 and related strains predicted by antiSMASH ${ }^{\text {a }}$.

\begin{tabular}{|c|c|c|c|c|c|c|c|}
\hline Strains & Total & DAPG & PLT & PRN & NRPS & Bacteriocin & Others \\
\hline P. protegens FJKB0103 & 16 & 1 & 1 & 1 & 7 & 2 & 4 \\
\hline P. protegens Cab57 & 14 & 1 & 1 & 1 & 5 & 2 & 4 \\
\hline P. protegens $\mathrm{CHA} 0^{\mathrm{T}}$ & 14 & 1 & 1 & 1 & 5 & 3 & 3 \\
\hline P. protegens $\mathrm{H} 78$ & 15 & 1 & 1 & 1 & 5 & 3 & 4 \\
\hline P. protegens SN15-2 & 16 & 1 & 1 & 1 & 6 & 2 & 5 \\
\hline P. protegens FDAARG & 15 & 1 & 1 & 1 & 5 & 3 & 4 \\
\hline P. protegens Pf-5 & 15 & 1 & 1 & 1 & 5 & 3 & 4 \\
\hline P. saponiphila DSM $9751^{\mathrm{T}}$ & 14 & 1 & 0 & 0 & 4 & 3 & 6 \\
\hline P. cerasi $58^{\mathrm{T}}$ & 9 & 0 & 0 & 0 & 5 & 1 & 3 \\
\hline P. meliae CFBP $3225^{\mathrm{T}}$ & 9 & 0 & 0 & 0 & 5 & 0 & 4 \\
\hline P. congelans DSM $14939^{\mathrm{T}}$ & 9 & 0 & 0 & 0 & 7 & 0 & 2 \\
\hline P. tremae ICMP9151 ${ }^{\mathrm{T}}$ & 15 & 0 & 0 & 0 & 9 & 0 & 6 \\
\hline P. caspiana $\mathrm{FBF} 102^{\mathrm{T}}$ & 6 & 0 & 0 & 0 & 2 & 2 & 2 \\
\hline
\end{tabular}

a Clusters identified by antiSMASH 5.0 using the "Extra Features" settings; Abbreviations are as follows: 2,4-diacetylphloroglucinol, DAPG; pyrrolnitrin, PRN; pyoluteorin, PLT; non-ribosomal peptide synthase, NRPS; $\mathrm{T}$ means type strain.

\subsection{DAPG as the Anti-MRSA Compound in P. protegens FJKB0103}

To confirm the roles of several compounds, including DAPG, PRN, PLT, and orfamide in antibiotic activities, the genes phlD, prnA, pltA, and orf A deleted mutants were constructed, and their anti-MRSA activities were tested. The mutant $\triangle \mathrm{phlD}$ failed to inhibit MRSA growth, and the other mutants $\triangle \mathrm{prnA}, \Delta \mathrm{pltA}$, and $\triangle$ orfA had the same antibacterial activities as the wild-type strain FJKB0103 (Figure 2). To further evaluate the role of gene phlD on antibacterial ability and DAPG production, the $\triangle$ phlD complementary strain $\triangle$ phlD-C was constructed. The anti-MRSA ability of strain $\triangle$ phlD-C was restored to the same level as wild-type strain FJKB0103, and also DAPG production was restored (Figure 2), these data supported that the DAPG biosynthetic gene cluster was involved in anti-MRSA activity of P. protegens FJKB0103. The anti-MRSA activity of DAPG biosynthetic gene cluster products, including DAPG, MAPG, and PG, was confirmed by detecting the MIC values. The MIC values of DAPG of all S. aureus strains were lower than $10 \mu \mathrm{g} / \mu \mathrm{L}$, which were similar to those of vancomycin, but the MIC of both MAPG and PG were higher than $128 \mu \mathrm{g} / \mu \mathrm{L}$ (Table 4 ). Moreover, after $18 \mathrm{~h}$, the bacterial suspension decreased that deal with DAPG of the MBC, $2 \mathrm{MBC}$, and $4 \mathrm{MBC}$ level (Figure 3), indicating that the bacterial cells were lysed. All these data showed that DAPG was the anti-MRSA compound in P. protegens FJKB0103.

\subsection{DAPG Affected the Biofilm Formation of S. aureus}

Knowing that DAPG inhibits the biofilm formation of Bacillus subtilis at the subinhibitory level [28], we wanted to ask if DAPG affected the biofilm formation of S. aureus. As biofilm formation was regulated by QS, which was related to bacterial growth [29], the subinhibitory level that did not affect the $S$. aureus strain growth was selected for detecting the effect on biofilm formation. At a subinhibitory level of DAPG, the biofilm formation of S. aureus Sta24-1 was decreased compared with that without DAPG (Figures 4 and S3), which was consistent with data from previous studies $[9,28]$. These data supported the idea that DAPG affected $S$. aureus strains by both antibiotic ability and repression of biofilm formation. 
A

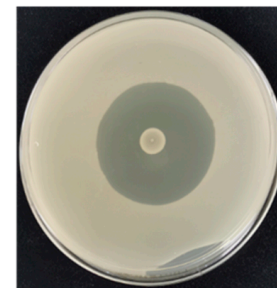

FJKB0103

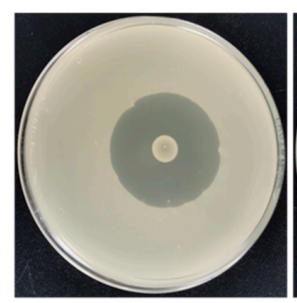

$\Delta \mathrm{pltA}$

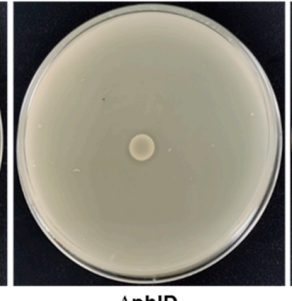

$\triangle$ phID

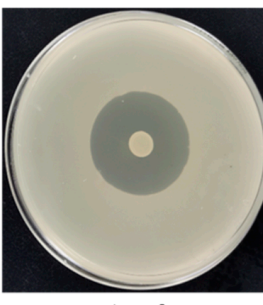

$\Delta$ prnA

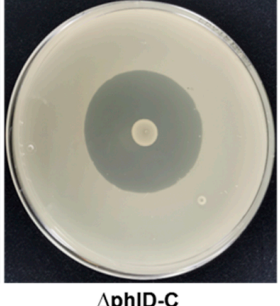

$\triangle$ phID-C

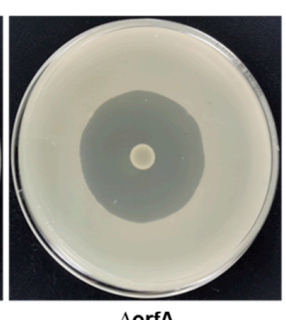

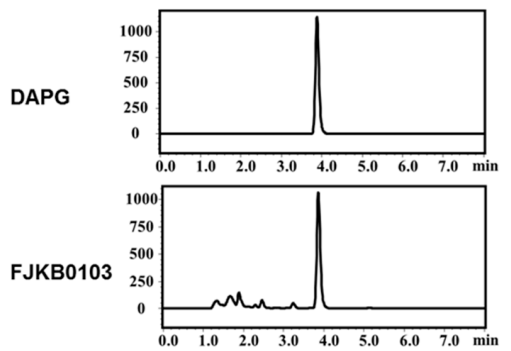

$\Delta$ phID

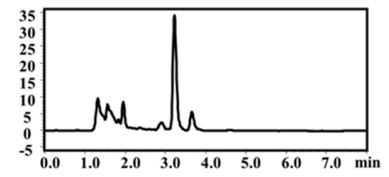

$\Delta$ phID-C

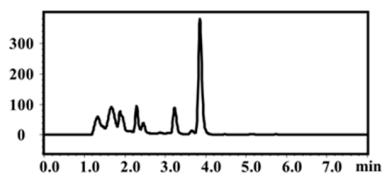

Figure 2. (A) Antimicrobial activity of P. protegens FJKB0103 and its mutants. For antibacterial assays, $5 \mu \mathrm{L}$ of overnight culture of $P$. protegens FJKB0103 and its mutants were dropped onto the PDA plates center. After inoculating at $28{ }^{\circ} \mathrm{C}$ for 2 days, the LB mixed with $10 \%$ saturated S. aureus Sta24-1 was poured on the PDA plates, inoculated at $37^{\circ} \mathrm{C}$ for $12 \mathrm{~h}$. (B) HPLC analysis of 2,4-DAPG production of $P$. protegens FJKB0103 and its mutants in KB medium. FJKB0103, the wild-type strain of P. protegens FJKB0103; $\Delta$ phlD, the phlD gene deleted mutant of P. protegens FJKB0103; $\Delta$ phlD-C, the complementary strain of $\triangle$ phlD with pRK-phlACBD.

Table 4. The minimum inhibitory concentration (MIC) and minimum bactericidal concentration (MBC) of DAPG, MAPG, PG, and vancomycin on S. aureus strains.

\begin{tabular}{ccccccccc}
\hline \multirow{2}{*}{ Strains } & \multicolumn{4}{c}{ MIC $(\mu \mathrm{g} / \mathrm{mL})$} & \multicolumn{3}{c}{ MBC $(\mu \mathrm{g} / \mathrm{mL})$} \\
\cline { 2 - 9 } & DAPG & MAPG & PG & Vancomycin & DAPG & MAPG & PG & Vancomycin \\
\hline S. aureus ATCC25293 & 4 & $>128$ & $>128$ & 2 & 32 & $>128$ & $>128$ & 32 \\
S. aureus ATCC29213 & 4 & $>128$ & $>128$ & 2 & 32 & $>128$ & $>128$ & 16 \\
S. aureus Sta403 & 4 & $>128$ & $>128$ & 2 & 16 & $>128$ & $>128$ & 16 \\
S. aureus Sta24-1 & 4 & $>128$ & $>128$ & 1 & 32 & $>128$ & $>128$ & 32 \\
\hline
\end{tabular}

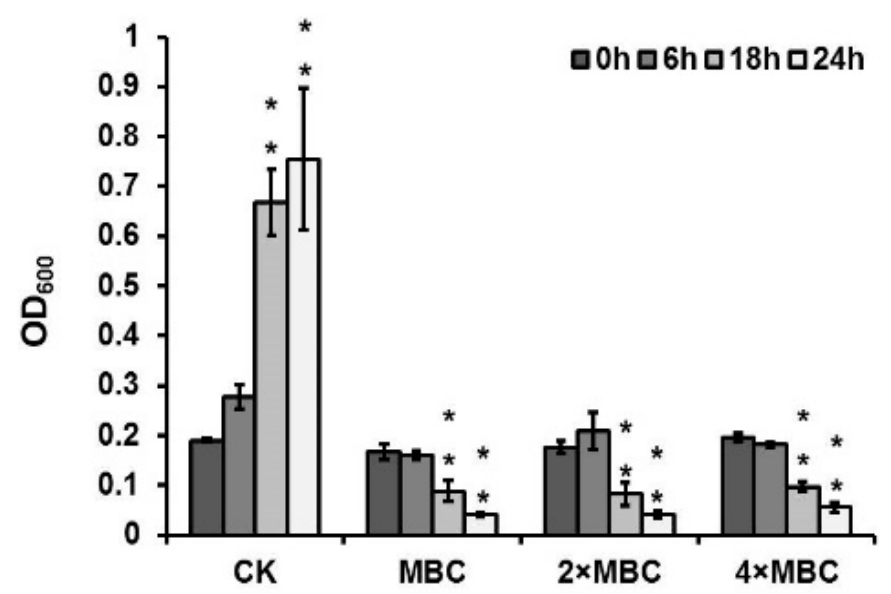

Figure 3. Bacteriolytic activity of DAPG against $S$. aureus Sta24-1 at $1 \times \mathrm{MBC}(32 \mu \mathrm{g} / \mathrm{mL}), 2 \times \mathrm{MBC}$ $(64 \mu \mathrm{g} / \mathrm{mL})$ and $4 \times \mathrm{MBC}(128 \mu \mathrm{g} / \mathrm{mL})$. The S. aureus Sta24-1 was exposed to DAPG with different concentrations at the early stage of exponential growth, and the negative control (CK) was exposed to methanol. The optical density $\left(\mathrm{OD}_{600}\right)$ was measured at $0,6,18$, and $24 \mathrm{~h}$ after incubation with DAPG. Mean values of three replicates are given, and error bars indicate standard error. ${ }^{* *}$ indicates $p<0.01$. 


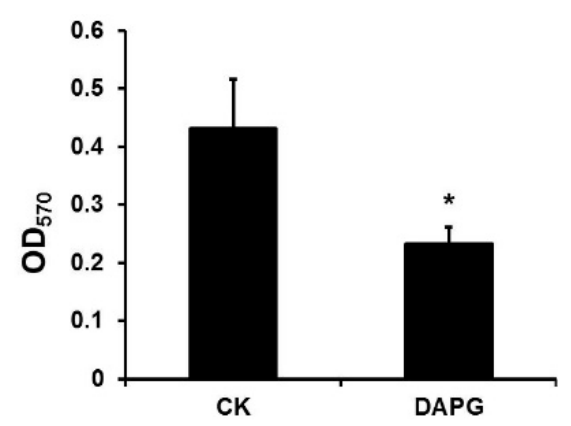

Figure 4. DAPG inhibited the biofilm formation of S. aureus Sta24-1. S. aureus Sta24-1 was incubated in $3 \mathrm{~mL}$ TSB glass tubes with $0.125 \mu \mathrm{g} / \mathrm{mL}$ DAPG or methanol (CK). Biofilms were stained with $0.1 \%$ crystal violet and quantified at $570 \mathrm{~nm}$. Mean values of three replicates are given, and error bars indicate standard error. * indicates $p<0.05$.

\section{Discussion}

Pseudomonas species produce diverse secondary metabolites that have antibiotic ability. For example, DAPG, PLT, PRN, and NRPS belong to the broad-spectrum antibiotics that inhibit both bacteria and fungi, and mupirocin is used to control S. aureus, particularly methicillin-resistant $S$. aureus (MRSA), when other antibiotics are ineffective [30]. Phenazine-1-carboxamide and 1-acetyl-beta-carboline produced by Pseudomonas species also have an inhibitory effect against MRSA [31,32]. The strain FJKB0103 has 16 metabolite biosynthetic gene clusters, including DAPG and PLT, that are conversed in P. protegens species [26]. PG, an intermediate in DAPG biosynthesis, has a concentration-dependent influence on the expression of PLT biosynthetic genes and the production of PLT [33]. In our study, the mutant $\triangle$ phlD failed to inhibit MRSA growth completely, but the deletion of $p l t A$ involved in PLT biosynthesis did not affect the anti-MRSA ability, indicating that the DAPG biosynthetic gene cluster participates in the anti-MRSA ability of P. protegens FJKB0103. Furthermore, the MRSA strains were resistant to MAPG and PG, but sensitive to DAPG, which was in correspondence with previous reports that DAPG is more active than MAPG and PG [34]. Having said all above, DAPG is the anti-MRSA compound in P. protegens FJKB0103.

Pseudomonas species could appear in different environments, including the soil, plant surfaces, and insects, and acquire more than one gene cluster conferring antibiotic biosynthesis during the evolutionary progress. The several antibiotics produced by one strain might be beneficial to its competition with certain competitors and predators or occupation of specific habitats [6]. For example, nunamycin plays the role primarily in Rhizoctonia solani AG3 mycelial growth inhibition, whereas nunapeptin was essential for Pythium aphanidermatum inhibition in P. fluorescens In5 [35], and cyclic lipodepsipeptides WAP-8294A is the antibacterial compound, while HSAF (dihydromaltophilin) inhibits the fungal growth in Lysobacter enzymogenes OH11 [36]. P. protegens FJKB0103 has 16 BGCs (Tables 3 and S4), which might be related to its special environmental niche [37]. DAPG produced by P. protegens FJKB0103 was the major antibacterial and antifungal compound (Figures 2 and S4), and orfamide participated in the swarming motility of P. protegens FJKB0103 (Figure S5), however, the roles of most of the multiple secondary metabolites with diverse chemical properties produced by $P$. protegens FJKB0103 remain unclear, which will be subject to future study.

Antibiotics play a significant role in biofilm formation, and most antibiotics tend to stimulate biofilm formation at subinhibitory concentrations. For example, subinhibitory concentrations of aminoglycoside antibiotics induce biofilm formation in P. aeruginosa and Escherichia coli [38]. DAPG stimulates biofilm formation in Azospirillum brasilense Sp245-Rif at a subinhibitory concentration [39], whereas polymyxin B had no effect on biofilm formation of P. aeruginosa and Escherichia coli [38]. By contrast, the biofilm formation was reduced when DAPG at a subinhibitory concentration was applied to Bacillus subtilis NCIB3610 and MRSA (Figure 3) [28]. Antibiotics might act as signal molecules at subinhibitory concen- 
trations, and the effect on biofilm formation can differ between antibiotic types and the strains [40].

DAPG might be a good alternative for controlling infections caused by MRSA: DAPG could lyse the bacteria and inhibit the growth at high concentrations, and represses biofilm formation at low concentrations $[9,25,41,42]$. Additionally, the MIC values of DAPG are similar to those of vancomycin (Table 4), which is the common antibiotic used to control MRSA infections. Furthermore, DAPG was not acutely toxic to mice when administered orally at single doses of up to $100 \mathrm{mg} / \mathrm{kg}$ [41], but the toxicity of DAPG by injection, absorbability in the digestive tract, and other pharmacodynamic properties remain unknown, thus there is more work needed for its application.

Supplementary Materials: The following supporting information can be downloaded at: https: / / www.mdpi.com/article/10.3390/microorganisms10020315/s1, Figure S1: (A) Anti-MRSA activity of strains isolated from rhizosphere. (B) Antibacterial activity of strain FJKB0103; Figure S2: (A) Neighbour-joining phylogenetic tree based on 16S rRNA gene sequences showing the relationship of strain FJKB0103 to the closely related strains. (B) Neighbour-joining phylogenetic tree based on MLSA (16S rRNA, gyrB, rpoD and $r p o B$ ) showing the relationship of strain FJKB0103 to the closely related strains; Figure S3: The growth curves of S. aureus Sta24-1 with DAPG at different concentrations; Figure S4: Antifungal activity of P. protegens FJKB0103 and its mutants against Rhizoctonia solani; Figure S5: The swarming motility of $P$. protegens FJKB0103 and its mutants on soft $(0.3 \% w / v)$ LB agar plates; Table S1: Strains and plasmids used in this study; Table S2: Primers used in this study; Table S3: Biochemical characteristics of strain P. protegens $\mathrm{FJKB} 0103$ and P. protegens $\mathrm{CHA} 0^{\mathrm{T}}$; Table S4: Secondary metabolite and antibiotic gene clusters in P. protegens FJKB0103 predicted by antiSMASH.

Author Contributions: Conceptualization, H.Z., X.X. and Q.W.; methodology, H.Z.; software, H.Z.; resources, L.Y., Y.L., S.W. and M.C.; writing-original draft preparation, H.Z.; writing-review and editing, H.Z., L.L., Q.G., X.X., J.Z. and Q.W.; supervision, Q.W.; funding acquisition, H.Z., L.L. and Q.W. All authors have read and agreed to the published version of the manuscript.

Funding: This research was funded by the National Key R\&D Program of China (2021YFA0910200), GDAS' Project of Science and Technology Development (2020GDASYL-20200103020), National Natural Science Foundation of China (Grant Number 32001812), Key Laboratory of Guangdong Province (2020B121201009).

Data Availability Statement: The accession numbers of the 16S rRNA gene sequence and the draft genome sequence in GenBank are OL377853 and JAJQXB000000000, respectively.

Conflicts of Interest: The authors declare no conflict of interest.

\section{References}

1. Tong, S.Y.C.; Davis, J.S.; Eichenberger, E.; Holland, T.; Fowler, V.G. Staphylococcus aureus Infections: Epidemiology, Pathophysiology, Clinical Manifestations, and Management. Clin. Microbiol. Rev. 2015, 28, 603-661. [CrossRef] [PubMed]

2. Chambers, H.F.; DeLeo, F.R. Waves of Resistance: Staphylococcus aureus in the Antibiotic Era. Nat. Rev. Microbiol. 2009, 7, 629-641. [CrossRef] [PubMed]

3. Jevons, M.P. “Celbenin"—Resistant Staphylococci. Br. Med. J. 1961, 1, 124-125. [CrossRef]

4. Witte, W. Antibiotic Resistance in Gram-Positive Bacteria: Epidemiological Aspects. J. Antimicro. B Chemoth. 1999, 44, 1-9. [CrossRef]

5. Craft, K.M.; Nguyen, J.M.; Berg, L.J.; Townsend, S.D. Methicillin-Resistant Staphylococcus aureus (MRSA): Antibiotic-Resistance and the Biofilm Phenotype. Med. Chem. Commun. 2019, 1, 1231-1241. [CrossRef]

6. Gross, H.; Loper, J.E. Genomics of Secondary Metabolite Production by Pseudomonas spp. Nat. Prod. Rep. 2009, 26, 1408-1446. [CrossRef]

7. Iessy, A.; Filion, M. Phloroglucinol Derivatives in Plant-Beneficial Pseudomonas spp.: Biosynthesis, Regulation, and Functions. Metabolites 2021, 11, 182.

8. Gleeson, O.; Gara, F.O.; Morrissey, J.P. The Pseudomonas fluorescens Secondary Metabolite 2,4 Diacetylphloroglucinol Impairs Mitochondrial Function in Saccharomyces cerevisiae. Anton. Leeuw. Int. J. G 2010, 97, 261-273. [CrossRef]

9. Julian, W.T.; Vasilchenko, A.V.; Shpindyuk, D.D.; Poshvina, D.V.; Vasilchenko, A.S. Bacterial-Derived Plant Protection Metabolite 2,4-Diacetylphloroglucinol: Effects on Bacterial Cells at Inhibitory and Subinhibitory Concentrations. Biomolecules 2021, 11, 13 [CrossRef] 
10. De Souza, J.T.; Arnould, C.; Deulvot, C.; Lemanceau, P.; Gianinazzi-Pearson, V.; Raaijmakers, J.R. Effect of 2,4-Diacetylphloroglucinol on Pythium: Cellular Responses and Variation in Sensitivity Among Propagules and Species. Biol. Control. 2003, 93, 966-975. [CrossRef]

11. Almario, J.; Bruto, M.; Vacheron, J.; Prigent-Combaret, C.; Moënne-Loccoz, Y.; Muller, D. Distribution of 2,4-Diacetylphloroglucinol Biosynthetic Genes among the Pseudomonas spp. Reveals Unexpected Polyphyletism. Front. Microbiol. 2017, 8, 1218. [CrossRef] [PubMed]

12. Achkar, J.; Xian, M.; Zhao, H.; Frost, J.W. Biosynthesis of Phloroglucinol. J. Am. Chem. Soc. 2005, 127, 5332-5333. [CrossRef] [PubMed]

13. Pavkov-Keller, T.; Schmidt, N.G.; Zadlo-Dobrowolska, A.; Kroutil, W.; Gruber, K. Structure and Catalytic Mechanism of a Bacterial Friedel-Crafts Acylase. Chembiochem 2019, 20, 88-95. [CrossRef] [PubMed]

14. Abbas, A.; McGuire, J.E.; Crowley, D.; Baysse, C.; Dow, M.; O'Gara, F. The Putative Permease PhlE of F113 Has a Role in 2,4-Diacetylphloroglucinol Resistance and in General Stress Tolerance. Microbiology 2004, 150, 2443-2450. [CrossRef]

15. Abbas, A.; Morrissey, J.P.; Marquez, P.C.; Sheehan, M.M.; Delany, I.R.; O'Gara, F. Characterization of Interactions between the Transcriptional Repressor PhlF and Its Binding Site at the phlA Promoter in Pseudomonas fluorescens F113. J. Bacteriol. 2002, 184, 3008-3016. [CrossRef]

16. Yan, X.; Yang, R.; Zhao, R.X.; Han, J.T.; Jia, W.J.; Li, D.Y.; Wang, Y.; Zhang, N.; Wu, Y.; Zhang, L.Q.; et al. Transcriptional Regulator PhlH Modulates 2,4-Diacetylphloroglucinol Biosynthesis in Response to the Biosynthetic Intermediate and End Product. Appl. Environ. Microbiol. 2017, 83, e01419-17. [CrossRef]

17. Wu, S.; Huang, J.H.; Zhang, F.; Wu, Q.P.; Zhang, J.M.; Pang, R.; Zeng, H.Y.; Yang, X.Y.; Chen, M.T.; Wang, J.; et al. Prevalence and Characterization of Food-Related Methicillin-Resistant Staphylococcus aureus (MRSA) in China. Front. Microbiol. 2019, 10, 304. [CrossRef]

18. Tamura, K.; Peterson, D.; Peterson, N.; Stecher, G.; Nei, M.; Kumar, S. MEGA5: Molecular Evolutionary Genetics Analysis Using Maximum Likelihood, Evolutionary Distance, and Maximum Parsimony Methods. Mol. Biol. Evol. 2011, 28, $2731-2739$. [CrossRef]

19. Lee, I.; Kim, Y.O.; Park, S.C.; Chun, J. OrthoANI: An Improved Algorithm and Software for Calculating Average Nucleotide Identity. Int. J. Syst. Evol. Micr. 2016, 66, 1100-1103. [CrossRef]

20. Meier-Kolthoff, J.P.; Auch, A.F.; Klenk, H.P.; Goker, M. Genome Sequence-Based Species Delimitation with Confidence Intervals and Improved Distance Functions. BMC Bioinformatis 2013, 14, 60. [CrossRef]

21. Blin, K.; Shaw, S.; Steinke, K.; Villebro, R.; Ziemert, N.; Lee, S.Y.; Marnix, M.H.; Weber, T. antiSMASH 5.0: Updates to the secondary Metabolite Genome Mining Pipeline. Nucleic Acids Res. 2019, 47, W81-W87. [CrossRef] [PubMed]

22. Ahmadzadeh, M.; Tehrani, A.S. Evaluation of fluorescent Pseudomonads for Plant Growth Promotion, Antifungal Activity against Rhizoctonia solani on Common Bean, and Biocontrol Potential. Biol. Control 2009, 48, 101-107. [CrossRef]

23. CLSI. Performance Standards for Antimicrobial Susceptibility Testing, 30th ed.; CLSI supplement M100; Clinical and Laboratory Standards Institute: Wayne, PA, USA, 2020.

24. Abdel-Hamid, M.; Romeih, E.; Saporito, P.; Osman, A.; Mateiu, R.V.; Mojsoska, B.; Jenssen, H. Camel Milk Whey Hydrolysate Inhibits Growth and Biofilm Formation of Pseudomonas aeruginosa PAO1 and Methicillin-Resistant Staphylococcus aureus. Food Control. 2020, 11, 107056. [CrossRef]

25. Kamei, Y.; Isnansetyo, A. Lysis of Methicillin-Resistant Staphylococcus aureus by 2,4-Diacetylphloroglucinol Produced by Pseudomonas sp. AMSN Isolated from a Marine Alga. Int. J. Antimicrob. A G 2003, 21, 71-74. [CrossRef]

26. Zhao, H.; Liu, Y.P.; Zhang, L.Q. In silico and Genetic Analyses of Cyclic Lipopeptide Synthetic Gene Clusters in Pseudomonas sp. 11K1. Front. Microbiol. 2019, 10, 544. [CrossRef]

27. Olorunleke, F.E.; Kieu, N.P.; De Waele, E.; Timmerman, M.; Ongena, M.; Hofte, M. Coregulation of the Cyclic Lipopeptides Orfamide and Sessilin in the Biocontrol Strain Pseudomonas sp. CMR12a. MicrobiologyOpen 2017, 6, e4995. [CrossRef]

28. Powers, M.J.; Sanabria-Valentín, E.; Bowers, A.A.; Shank, E.A. Inhibition of Cell Differentiation in Bacillus subtilis by Pseudomonas protegens. J. Bacteriol. 2015, 197, 2129-2138. [CrossRef]

29. Yarwood, J.M.; Douglas, J.; Bartels, D.J.; Volper, E.M.; Greenberg, E.P. Quorum Sensing in Staphylococcus aureus Biofilms. J. Bacteriol. 2004, 186, 1838-1850. [CrossRef]

30. Gurney, R.; Thomas, C.M. Mupirocin: Biosynthesis, Special Features and Applications of an Antibiotic from a Gram-Negative Bacterium. Appl. Microbiol. Biot. 2011, 90, 11-21. [CrossRef]

31. Cardozo, V.F.; Oliveira, A.G.; Nishio, E.K.; Perugini, M.R.E.; Andrade, C.; Silveira, W.D.; Durán, N.; Andrade, G.; Kobayashi, R.; Nakazato, G. Antibacterial Activity of Extracellular Compounds Produced by a Pseudomonas Strain against Methicillin-Resistant Staphylococcus aureus (MRSA) Strains. Ann. Clin. Microb. Anti. 2013, 12, 12. [CrossRef]

32. Lee, D.S.; Eom, S.H.; Jeong, S.Y.; Shin, H.J.; Je, J.Y.; Lee, E.W.; Chung, Y.H.; Kim, Y.M.; Kang, C.K.; Lee, M.S. Anti-MethicillinResistant Staphylococcus aureus (MRSA) Substance from the marine Bacterium Pseudomonas sp. UJ-6. Environ. Toxicol. Phar. 2013, 35, 171-177. [CrossRef] [PubMed]

33. Yan, Q.; Philmus, B.; Chang, J.; Loper, J.E. Novel Mechanism of Metabolic Co-Regulation Coordinates the Biosynthesis of Secondary Metabolites in Pseudomonas protegens. eLife 2017, 6, e22835. [CrossRef] [PubMed]

34. Islam, M.T.; von Tiedemann, A. 2,4-Diacetylphloroglucinol Suppresses Zoosporogenesis and Impairs Motility of Peronosporomycete Zoospores. World J. Microb. Biot. 2011, 27, 2071-2079. [CrossRef] [PubMed] 
35. Michelsen, C.F.; Watrous, J.; Glaring, M.A.; Kersten, R.; Koyama, N.; Dorrestein, P.C.; Stougaard, P. Nonribosomal Peptides, Key Biocontrol Components for Pseudomonas fluorescens In5, Isolated from a Greenlandic Suppressive Soil. mBio 2015, 6, e00079-15. [CrossRef]

36. Wang, Y.; Qian, G.; Liu, F.; Li, Y.Z.; Shen, Y.; Du, L. Facile Method for Site-specific Gene Integration in Lysobacter enzymogenes for Yield Improvement of the Anti-MRSA Antibiotics WAP-8294A and the Antifungal Antibiotic HSAF. ACS Synth. Biol. 2013, 2 670-678. [CrossRef]

37. Chiellini, C.; Lombardo, K.; Mocali, S.; Miceli, E.; Fani, R. Pseudomonas Strains Isolated from Different Environmental Niches Exhibit Different Antagonistic Ability. Ethol. Ecol. Evol. 2019, 31, 399-420. [CrossRef]

38. Hoffman, L.R.; D’Argenio, D.A.; MacCoss, M.J.; Zhang, Z.; Jones, R.A.; Miller, S.I. Aminoglycoside Antibiotics Induce Bacterial Biofilm Formation. Nature 2005, 436, 1171-1175. [CrossRef]

39. Combes-Meynet, E.; Pothier, J.F.; Moënne-Loccoz, Y.; Prigent-Combaret, C. The Pseudomonas Secondary Metabolite 2,4-Diacetylphloroglucinol Is a Signal Inducing Rhizoplane Expression of Azospirillum Genes Involved in Plant-Growth Promotion. Mol. Plant Microbe. Interact. 2011, 24, 271-284. [CrossRef]

40. Romero, D.; Traxler, M.F.; López, D.; Kolter, R. Antibiotics as Signal Molecules. Chem. Rev. 2011, 111, 5492-5505. [CrossRef]

41. Lord, J.; Gikonyo, A.; Miwa, A.; Odoi, A. Antimicrobial Resistance among Enterobacteriaceae, Staphylococcus aureus, and Pseudomonas spp. Isolates from clinical Specimens from a Hospital in Nairobi, Kenya. PeerJ 2021, 9, e11958. [CrossRef]

42. Isnansetyo, A.; Cui, L.Z.; Hiramatsu, K. Antibacterial Activity of 2,4-Diacetylphloroglucinol Produced by Pseudomonas sp. AMSN Isolated from a Marine Alga, against Vancomycin-Resistant Staphylococcus aureus. Int. J. Antimicrob. A G 2003, 22, 545-547. [CrossRef] 